\title{
Advancing Interprofessional Collaboration in Nursing Education
}

\begin{abstract}
By Joanne S. Hebert
The purpose of this qualitative case study was to describe and understand the process of nurse educators teaching interprofessional collaboration to student nurses in a Louisiana pre-licensure baccalaureate-nursing program. Components of the theoretical framework for the study included Ray's theory of bureaucratic caring, complexity theory, and transformational learning theory. As interprofessional collaboration is gaining momentum in the healthcare environment, nurse educators can be key players in a teaching-learning dynamic that encourages positive patient outcomes and a changing healthcare paradigm. Findings indicated the majority of nurse educators describe their role in preparing students for interprofessional collaborative practice using classroom, clinical and simulation teaching strategies. Clinical environments were most often described as settings in which students gain the most knowledge through actual participation with the interprofessional team. Findings from this study indicate a need for curriculum changes and enhanced faculty development programs on interprofessional collaboration in nursing education. Nurse educators and nursing programs can learn from this case study to develop teaching strategies to promote conducive interprofessional collaborative learning environments aiding students in the preparation for professional nursing practice.
\end{abstract}

Keywords: Collaboration, Healthcare reform, Interprofessional education competencies, Nursing education.

\section{Introduction}

Interprofessional collaboration is a healthcare initiative where teamwork is required to meet the increasingly complex needs of patients. Teaching student nurses to learn and practice interprofessional collaboration is the topic of this qualitative research case study. Compatibility in a collaborative process means healthcare professionals will work collectively to enable each other to accomplish more together than if they were working on their own. As interprofessional collaboration is gaining momentum in the healthcare reform environment, nurse educators can be key players in a teaching-learning dynamic that encourages positive patient outcomes and a changing healthcare paradigm. This case study gathered perspectives about preparing competent students for interprofessional collaborative practice from nurse educators in a Louisiana pre-licensure, baccalaureate-nursing program. Understanding the perspectives of nurse educators regarding interprofessional collaboration is crucial in developing learning opportunities for graduate baccalaureate students to practice effectively.

\footnotetext{
${ }^{*}$ Nursing Faculty, College of Nursing and Health Sciences, Southeastern Louisiana University USA.
} 


\section{Background, Context, and Theoretical Framework}

The Tri-Council for Nursing (2010) includes the American Association of Colleges of Nursing (AACN), American Nurses Association (ANA), American Organization of Nurse Executives (AONE), and the National League for Nursing (NLN). The Tri-Council position statement (2010) calls for collaborative action in support of the Institute of Medicine's (IOM) Future of Nursing Report (2010). Rationale for basing the future of nursing on the IOM report was given, along with NLN's affirmation as a privileged participant with the IOM and Robert Wood Johnson Foundation (RWJF) in advancing nursing education's academic progress while improving the nation's health. According to the Tri-Council for Nursing position statement (2010), the IOM, established in 1970, is a body of interdisciplinary members who advise the nation on health issues impacting Americans.

In 2006, RWJF selected the Institute of Healthcare Improvement (IHI) to manage the national initiative of New Health Partnerships: Improving Care by Engaging Patients. IHI launched innovative work on the Triple Aim (2007) framework to 1) improve the patient experience of care (including quality and satisfaction), 2) improve the health of populations, and 3) reduce the per capita cost of healthcare. In the United States, areas of health reform can be furthered and strengthened by Triple Aim models, including accountable care organizations (ACOs), bundled payments, patient-centered medical homes, and integration of information technology (IHI 2015). Penalties for avoidable events, such as hospital readmissions or infections are examples of quality and safety programs.

In 2007, RWJF funded the Quality and Safety Education for Nurses (QSEN) project, grounded in the IOM report, Health Professions Education: A Bridge to Quality (2003). The QSEN project focused on the IOM (2003) report's safety and quality contexts. QSEN's goal was to transform nursing education by changing the focus of nursing's professional identity. The QSEN advisory board identified 6 core quality and safety clinical competencies. The QSEN core competencies include (a) patient-centered care, (b) teamwork and collaboration, (c) evidence-based practice, (d) quality improvement, (e) safety, and (f) informatics. Using the competency definitions, Cronenwett et al. (2007) described performance statements of knowledge, skills, and attitudes (KSAs) for each competency to be developed during pre-licensure nursing education.

The IOM (2010) report clearly stated patients receive safer, higher quality care when health professionals work effectively in teams, communicate productively, and understand each other's roles. Realizing nurses are the largest group in the healthcare workforce, the Tri-Council for Nursing (2010) strongly endorsed the new IOM report. Therefore, nurses are in a position to lead the initiative to ensure safe, high quality care to a diverse patient population.

Embedded in current healthcare and nursing education reform literature are common themes related to competencies. There is a sense of urgency for all healthcare professions to implement interprofessional collaboration (IPC), 
interprofessional education (IPE) and interprofessional collaborative practice (ICP) in a patient-centered healthcare environment. In 2010, the passage of the Patient Protection and Affordable Care Act (ACA) caused healthcare providers and policy makers to realize with primary care physician and nursing shortages, increased collaboration, and teamwork among health professions is necessary to provide care for an increasingly aging population with multiple comorbid chronic conditions.

Recently, the Health Resources and Services Administration (HRSA), Josiah Macy Jr. Foundation (JMJF), RWJF, and American Board of Internal Medicine Foundation joined forces with the Interprofessional Education Collaborative (IPEC). The expert interprofessional panel developed core competencies for interprofessional collaborative practice. Schmitt (2011) described interprofessional competencies as behavioral demonstrations of an integrated set of knowledge, skills, and attitudes for working together across the professions, with patients, families, communities, and populations to improve health outcomes in specific care contexts. Schmitt's (2011) model illustrates the four IPEC competency domains (a) value and ethics, (b) roles and responsibilities, (c) interprofessional communication, (d) interprofessional teamwork and team-based care. From these domains, the four core competencies (a) patient-centered care, (b) quality improvement,(c) evidencebased practice, and (d) informatics emerged. IPEC core competencies, also referred to as AACN competencies in this study, were addressed in face-to-face interviews with this study's participants.

\section{Theoretical Framework}

Components of the theoretical framework for this research study included Ray's theory of bureaucratic caring (RTBC), complexity theory, and transformational learning theory. Interrelated concepts, assumptions and generalizations of the blended theories appropriately support the study's foundation and efficacy of findings.

Ray (1998) discussed the complexity of nursing science, concluding, "Nursing may well be on its way to discovering the unified theory that complexity scientists are searching for by its knowledge of human-environment integrality, caring, relatedness, belongingness, and interconnectedness" (p. 93). An objective of this research study was to help nurse educators understand the changing roles they face, and how teaching interprofessional collaborative practice in an ever-changing, complex healthcare environment, is necessary to reform and transform our current patient-care system.

Blending complexity theory and transformational learning theory with RTBC (a nursing theory grounded in holistic nursing), a framework was posed for understanding the phenomena of interprofessional collaboration from an organizational culture and educational perspective. Ray's nursing theory, relates to the political, economic, sociocultural environment of complex health systems, and sheds light on the issues identified in advancing interprofessional collaboration in nursing education. Rafferty (as cited by Ray 1998) calls for 
nursing and other disciplines to form strong coalitions. Rafferty suggested nursing should collaborate with sociologists and economists to shape an agenda for health services research and transformation of health policy.

Ray (1998) goes on to explain the place of complexity theory in nursing's theories and science, "Nursing theorists of the last quarter century have reconstructed the science of nursing in unique and creative ways to illuminate the depth and complexity of nursing" (p. 92). Hidalgokehoe (2012) discussed how complexity theory supports the often devalued, intangible, task-oriented nursing care seen in productivity-based organizations. RTBC and complexity theory warrant aspects of transformation, and of change in the nursing profession. With the advent of Magnet Program recognition, nursing excellence requires nursing theories and practice models to be integrated into patient-centered care for best practice and evidence based practice (Turkel et al. 2012).

From a nursing education perspective, the issues of a healthcare delivery system experiencing reformation and transformation may be taught through the lens of change using the theory of transformational learning. Taylor (as cited in Merriam et al. 2007) describes the lenses of transformational learning coming from two loci of learning perspectives, the individual, and the socio-cultural. A well-known transformational sociocultural approach is Freire's social emancipatory view (or advocacy-liberatory view) of transformational learning. The social-emancipatory philosophical framework was used for this study's data collection approach. The advocacy-liberatory framework indicates direct relevance to the concept of interprofessional collaboration as a reality dependent on social, political, and economic contexts (Lodico et al. 2010). An advocacy-liberatory framework was used to not only employ an inductive process for gathering information about teaching, but to be a social advocate for nurse educators by identifying the types of changes needed to educate student nurses in the current healthcare environment. While blending the three theoretical components of the study's framework, this study attempted to explain how nurse educators transform and integrate the complex concept of nursing and interprofessionality into the current bureaucratic caring work environment.

\section{Review of Literature Regarding Advancing Interprofessional Collaboration in Nursing Education}

Although AACN Essentials of Baccalaureate Education for Professional Nursing Practice were published in 2008, there is little literature to support nursing education's urgency to promote new teaching strategies that address IPC competencies. Areas of concern in nursing academia as it relates to IPC include: (a) terminology, (b) IPC in nursing academia, (c) IPC as it relates to healthcare reform, (d) evidence based interprofessional practice, and (e) measurement instruments and reported outcomes. 


\section{Terminology}

Current literature is emendated with research on the concept of IPC, but not specifically in nursing education. Advanced searches revealed much more research on how academia is implementing IPE programs. Research is not as evident in nursing as other healthcare professions, and many more studies were conducted in Canada, United Kingdom, and Australia, than the United States. For purposes of this research study, IPC as it relates to nursing education are discussed and defined using nurse scholars, national nursing organizations, and interprofessional foundations and organizations perspectives.

Alberto and Herth (2009) conducted an expansive literature review on the subject of IPC. They describe the term collaboration as a positive concept used interchangeably with teamwork and explain the roots of the word, co and laborare, combined in Latin mean "work together" (p. 2G). Henneman et al. (1995) as cited in Alberto and Hearth (2009) stated, "collaboration involves partnership characterized by mutual goals and commitments in which participants willingly become involved in planning and decision making" ( $\mathrm{p}$. 263). To accomplish collaboration, each individual discipline is required to understand and appreciate what each contributes to the whole (p. 2G). In more recent research, O'Brien (2013) gives an example of interprofessional collaboration as a doctor, nurse, physical therapist, occupational therapist, and pharmacist collaborating to implement an individualized plan of care to improve a patient's health status, emphasizing this is not a new concept in healthcare.

The national United States organization, Interprofessional Education Collaborative (IPEC) does not define interprofessional collaboration, but does define IPCP based on the WHO (2010) report, as "when multiple healthcare workers from different professional backgrounds work together with patients, families, caregiver(s), and communities to deliver the highest quality care" (p. 2). This definition was accepted as the definition of IPCP relevant to this research study.

\section{Interprofessional Collaboration in Nursing Academia}

Advancing nurse educators' scientific knowledge base in IPCP is the catalyst for developing a new generation of registered nurses with a new perspective of nursing. Stokowski (2011) reported the 1988 NLN report attempting to overhaul nursing education with nursing curriculum reform brought no change. During the next 25 years under the auspices of healthcare reform, random curricular changes were made without changing the educational paradigm. It was not until 2003 when NLN advocated for a transformation of nursing education, that innovative pedagogies were developed. Stokowski (2011) went on to explain, this was the beginning of a new approach, one that would move away from the "old and tired clinical placement model", to one relevant for increasing the community-centered, multidisciplinary patient-centered healthcare delivery system of the future (p. 
2). Stokowski (2011) discussed the FON initiative as a joint venture of the IOM and RWJF. Three national forums critically reviewed the nursing profession, one of which pinpointed nursing education. Bleich, Dean of Oregon Health and Science University School of Nursing led the forum. Stokowski discussed her conversation with Bleich. He related how "it is more than knowing how to perform tasks and procedures; it is how to be a more effective player on the healthcare team, navigating clinical systems. Healthcare organizations expect nurses to perform at a higher level, to participate and contribute to the quality and safety agenda of the organization" (Stokowski 2011 , p. 3). The FON education report established today's nursing curricula are outdated, in a content-laden, passive learning environment. Students continue to rotate through specialty units following a disease-oriented medical model, with clinical opportunities primarily in acute care settings, with emphasis on preparing students for their nursing board exams. The registered nurse licensure exam is a minimum standard, testing for minimum safe competency. The public wants optimal competency. Competency means higher-level skills that demonstrate the ability to master care management, and decision making under various clinical situations across all healthcare settings. Another goal set by the FON initiative was to increase the proportion of baccalaureate prepared nurses to $80 \%$ by 2020 (Stokowski 2011).

One objective of the IOM and RWJF (2011) initiative is IPE, which requires IPC to coordinate care effectively. Poor communication and lack of respect between healthcare professions leads to poor patient outcomes. O'Brien (2013) noted that in 2003 the Joint Commission for Accreditation of Hospitals (JCAHO) reported communication failures among team members are a contributing factor in $60 \%$ of sentinel events. O'Brien (2013) added a few years later, the $2005 \mathrm{JCAHO}$ report found "communication failures were the leading root cause of all sentinel events" (p. 5).

\section{Interprofessional Collaboration as it Relates to Healthcare Reform}

O'Brien (2013) reviewed the history of IPC noting it is not a new concept. Coggeshall, a 1928 physician, interested in advancing medical progress through education suggested in 1965, the concept of medicine as a single discipline be replaced by the concept of health professions working together to maintain and increase the health of society as well as the individual. By 1970, the Lysaught Report released by the national commission for the Study of Nursing and Nursing Education endorsed the idea that nurses and physicians work together to improve patient care.

Garity (2005) discussed the relationship of the ANA Code of Ethics for Nurses with Interpretative Statements to nurses' collaborative efforts. Garity (2005) suggested "the Code as a thoughtful framework for nurses to evaluate their collaborative skills" (p. 1). The IOM (1972) report recommended the advancement in health teams. The AACN (2002) published a white paper, which highlighted interprofessional collaboration. Alberto and Herth (2009) report "NLN held an Education Summit in 2003 focusing on IPE, which lead to 
a position statement, Innovation in Nursing Education: A Call to Reform, followed by standards known as Hallmarks of Excellence, designed to define excellence in ten aspects of nursing education" (p. 5G). The AACN (2005) Annual Report included several references to productive collaboration. In addition, the AACN 2005 national conference focused on models of beneficial collaborative efforts among health professions. Varkey et al. (2006) conducted an interdisciplinary 4-week experiential quality improvement rotation at a major academic medical center. They noted a curriculum focused on disease and disease management is no longer adequate for the training of healthcare providers practicing in a milieu where medication errors, systems shortcomings, and practice scorecards are the norm. The focus of the project was outpatient medication reconciliation, a top JCAHO safety initiative.

With passage of the Patient Protection and Affordable Care Act (2010) changes in healthcare will be significant. The ACA identified care coordination and interprofessional care teams as important in improving health outcomes, preventing hospitalization, and reducing cost of care for older adults with chronic diseases. While rapid redesign of healthcare delivery, stimulated in part by the ACA, is occurring, missing is the ability to connect practice redesign with IPE reforms. Historically health professions education and practice has been siloed, perpetuating isolation of the professions. Making the important connection between IPE and collaborative practice will create an environment where all participants learn, all teach, all care, and all collaborate (JMJF 2013).

\section{Measurement Instruments and Reported Outcomes}

The Carnegie Foundation (2010) conducted a multiyear comparative study for the advancement of teaching professional education in the United States. The Foundation's report included findings by Benner et al. (2009). The American Association of Colleges of Nursing-Carnegie Foundation Nursing Education Study Survey Instrument (AACN-CFNESI) was administered between December 1, 2006 and March 5, 2007 to nurse faculty in the United States teaching pre-licensure undergraduate nursing programs.

Didion et al. (2013) reported "The Carnegie Foundation work found that too great a gap exists between what nurses learn in school and what is needed in practice. While nursing knowledge is becoming increasingly complex, it needs to focus in ways that are relevant, so that the transition from graduate to competent practitioners occurs quickly. Two major challenges face nursing educators: educate practitioners who will not only perform safe care but will also create safe systems, and do it in a way that minimizes the gap between education and practice" (p. 89). Profound changes in nursing practice include participating in interdisciplinary care team conferences, coordinating care, and delivering the highest level of quality care during the shortest length of inpatient stay. A slightly modified survey instrument (AACN-CFNESI 2) used in the Carnegie Foundation study was administered in this case study. 


\section{Review of Methodological Issues}

Despite numerous research studies and publications on IPC, there was no research to date using qualitative case study methodology in nursing education to understand how undergraduate baccalaureate nurse educators teach IPC. Although not a new concept, there is evidence demonstrating that some form of interprofessional, multidisciplinary, interdisciplinary, transdisciplinary collaboration, or teamwork has been successful in increasing patient satisfaction and patient outcomes.

Based on current literature, there are studies addressing faculty attitudes towards IPE. More quantitative studies on interprofessional collaboration were found. Thannhauser et al. (2010) found twenty-three quantitative instruments in their review of interprofessional literature. IPE models of care are becoming evident with nursing theoretical frameworks, but more testing and evaluation are necessary before they are integrated into curricula and evidence-based nursing education.

Literature findings reveal recommendations, endorsement, and support for IPC from health professions organizations including the IOM, RWJF, NLN, AACN, ANCC, IPEC, JMJF, Association of American Medical Colleges, American Association of Osteopathic Medicine, American Association of Colleges of Pharmacy, American Dental Education Association, and the Association of Schools of Public Health. The social sciences, physical and occupational therapy, are actively researching IPC as it relates to their professions. As evidenced by the literature review; there were many more international studies, especially in Canada and the United Kingdom. However, no qualitative case study research in nursing education was found. The methodology for this research study was supported by existing literature.

\section{Statement of the Problem}

The IOM (2010) report clearly stated patients receive safer, higher quality care when health professionals work effectively in teams, communicate productively, and understand each other's roles. Increased complexity of patient care requires interprofessional collaboration, as physicians are no longer able to meet the diversity of patient's needs independently. The JMJF (2013) recommended incorporating healthcare educational reform and practice redesign, emphasizing they not be developed in isolation.

Current literature identifies the need for new strategic nursing knowledge related to IPC. Interprofessional collaborative care is a requirement of registered nurses once in the practice setting. Benner et al. (2009) suggested integrating interprofessional concepts and competencies in the classroom and clinical practice settings as vital in teaching students to perform successfully in current complex, bureaucratic, interprofessional care environments. Therefore, the research problem underlying this case study is the gap in knowledge regarding the specific teaching strategies, processes, and behaviors used to 
educate future graduates to perform effectively in interprofessional healthcare environments. The central research question emerged in regards to the identified research problem.

\section{Research Questions}

The study focused on the central question: How do nurse educators in a prelicensure undergraduate baccalaureate-nursing program describe their role in preparing students for interprofessional collaborative care practices? Research sub-questions included:

1. How do these nurse educators incorporate the impact of health policy and government regulations in their teaching philosophy?

2. How do these nurse educators describe the barriers to teaching students to practice in a cost controlled healthcare environment?

3. How do these nurse educators teach students to understand their unique professional role and the role of other disciplines in the care of their patients?

4. How has the current interprofessional healthcare environment changed the lives of these nurse educators?

Adding sub-questions was a method to begin categorizing. Interview protocol included the following probing questions:

1. Tell me your understanding of interprofessional collaboration in nursing.

2. When you first learned of interprofessional collaboration in nursing education, what were your thoughts and concerns about teaching it?

3. Describe or give me some examples of how you teach interprofessional collaboration.

4. How has healthcare reform changed your teaching philosophy?

5. How do you address health policy and government regulations with students so they understand the impact on patient care?

6. Do you think interprofessional collaboration will affect healthcare reform? How?

7. Describe barriers you encounter when teaching students to practice in a cost effective healthcare environment.

8. How do you teach students to understand their role and the role of other disciplines in today's healthcare environment?

9. Do students learn QSEN or AACN competencies? If so, which level?

10. Do students understand the team approach to healthcare? How does this reflect in their care planning?

11. How has the interprofessional healthcare environment changed you as an educator? 


\section{Methodology}

The methodology for this research study was a qualitative case study, using Yin's (2009) model describing case study research as a linear but iterative process.

\section{Sample}

With the majority of nurse educators teaching in a pre-licensure baccalaureate-nursing program; the population for this study was narrowed and defined as those nurse educators teaching undergraduate, pre-licensure nursing students in one Louisiana nursing program.

Potential participants were purposefully selected due to the nature of the research questions. The focus of the study was to study nurse educators in natural teaching contexts. The logic and power of purposeful sampling comes with selecting information-rich cases for in-depth study. The goal of purposeful sampling was not to obtain a large and representative sample, but to select persons, places, or things that can provide the richest and most detailed information to help answer the research questions (Lodico et al. 2010). The setting of the study was selected because the research questions could best be answered in that setting. A purposeful sample represented the group to best answer the research question in the proposed setting (Yin 2009).

Potential study participants were full and part-time nurse educators teaching undergraduate pre-licensure nursing courses at a Louisiana college of nursing. The rationale for selecting this sample of participants was they were in a setting that best represents the phenomena studied in the research. Fifty-three potential participants were identified.

\section{Data Collection}

Yin (2009) pointed out characteristics a case study must possess. This case study complied with those characteristics:
a. focuses on in-depth examination of a bounded case,
b. seeks to set a case in its historical, social, and cultural context,
c. detail on physical setting and participants are presented, and
d. multiple data collection methods.

With these characteristics describing this case study, data collection methods included:
a. questions from an online survey,
b. semi-structured face-to-face interviews,
c. open conversation face-to-face interviews, and
d. participant observation. 
The online survey was imperative to gathering data to meet the second characteristic; set a case in its historical, social, and cultural context as well as the third characteristic; obtaining details on physical settings and participants. Yin (2009) addressed six sources of evidence for case study research; documentation, archival records, interviews, direct observation, participant observation, and physical artifacts. No single source of evidence has an advantage over the other. In fact, Yin suggested multiple sources highly complement each other and a good case study will use as many data sources as possible.

An advocacy-liberatory framework was used to not only employ an inductive process for gathering information about teaching, but to be a social advocate for nurse educators by identifying the types of changes needed to educate student nurses in our current healthcare environment. The focus is IPC concerning patient care. The objective of the qualitative method is to transform nursing education praxis by initiating critical reflection, problem posing, and dialogue to discover meaning and gain insight into IPC, emerging complex healthcare reform, and nursing education strategies (Merriam et al. 2007).

Guiding interview questions developed for semi structured and free conversation interviews were created as an interview protocol. Interview protocol guided the data collection in an organized, focused manner. Additional questions were probed for unexpected issues that emerged.

Features of careful observation included an explanation of the physical setting (classroom or clinical venue), a detailed description of the participants, individual and group dynamics during activities and group interactions, nonverbal communication and participant conversations with students, and finally researcher behavior (Lodico et al. 2010). Field notes were documented on the behavior and activities of individuals at the research site, followed by analysis of data for themes or issues. An observation protocol was developed specifically for this case study. Descriptive and reflective field notes were recorded to prevent bias in written description of researcher participantobservations.

\section{Instrumentation}

The Carnegie Foundation's instrument (AACN-CFNESI) was slightly modified (ACN-CFNESI2) with Benner's permission to address this case study's research questions. The scope of the study was narrowed, or bounded by asking for field test review of the modified survey instrument, AACNCFNESI2.

Survey data were collected over a 1-month period using the online webbased SurveyMonkey. Using the survey early in the data collection process generated information to begin analyzing participant characteristics and educational values. 


\section{Data Analysis}

Three data sources were analyzed in this study. Therefore, three sections describe each source, (a) survey findings, (b) face-to-face interview findings, and (c) participant-observation findings. Last, evidence from the multiple data sources were triangulated, identifying converging lines of inquiry.

Data collection and analysis involved researcher-participant collaboration to ensure the data accurately represented participant experiences. Data analysis and interpretation were inductive processes and ongoing throughout the study. The logic of this study's inductive approach was (a) data gathered, (b) openended questions asked and field notes recorded, (c) data analyzed to form themes, and (d) generalizations posed, linking them to the study's theoretical framework. Data were analyzed, prepared, and organized to limit influence on findings from researcher bias. Written, detailed reflective notes were another method used to prevent researcher bias.

Insights gained in early data collection guided future data collection. Audio-recorded face-to-face interviews were transcribed. Reading interview transcripts, reviewing observation notes and survey results detected themes and emerging patterns. Summaries of observation-participant data were interpreted in rich, thick descriptions. After a thorough review of the collected data, data saturation occurred and data collection concluded.

At this time, a qualitative data analysis expert was consulted. The researchers used NVivo9, the recommended qualitative data analysis software to complete analysis (QSR International Pty Ltd. 2010). NVivo enabled organization, analysis, and grouping of gathered data. A univariate basic descriptive analysis was conducted for the closed ended survey questions.

Coding the data into categories began. A standard thematic analysis was followed. Interview transcripts were input into the software and reviewed for common and/or different themes. The detailed participant observation transcripts were also input providing rich in-depth descriptions of participants, settings, and activities. Building themes from several codes allowed examination of guiding research sub-questions from the interview protocol. Verbatim text from the qualitative data was used to justify themes.

Generalizations were then made through the study's theoretical lens to describe how nurse educators explain their role as teachers of interprofessional collaboration. Lastly, multiple data sources were compared through triangulation. 
Findings

Emerging Themes from Online Survey

Data analysis of the online survey responses led to the emergence of the following themes:

1. Nurse educators teach their students through classroom activities, clinical settings, and the skills or simulation laboratory.

2. Nurse educators spend most of their work time teaching nursing students and conducting clinical instruction.

3. Graduating students are challenged in coordinating interprofessional collaborative care for individual patients that may minimize hospital stays.

4. Nurse educators believed that role-playing and encouraging students to interact with other professionals in the hospital are the teaching strategies needed to advance IPC in nursing education.

Survey findings laid the foundation from a historical perspective of the teaching/learning environment.

\section{Emerging Themes from Face-to-Face Interviews}

The findings of the data analysis of face-to-face interviews led to the emergence of the following major themes:

1. IPC means different professionals working together.

2. IPC is a team-based approach.

3. IPC is taught through case studies and analysis.

4. Health policies are always included when teaching and have not caused changes in teaching philosophy.

5. Lack of proper management of policies in hospitals.

6. No barriers to teaching students cost effective care.

7. Students do not learn QSEN or AACN competencies.

8. Educators emphasize and expose students to collaboration and teamwork.

9. Actual hands-on experiences are most used as teaching strategy for IPC care. (examples included participating in interprofessional/ multidisciplinary patient rounds, collaborating with nurses, respiratory therapist, dietician, pharmacist, case manager and social worker on patient discharge status and post hospitalization plans).

10. No major changes in the lives of nurse educators teaching in the interprofessional care environment. Nurse educators describe IPC as the old discharge planning model they have taught for years. 
A number of minor themes emerged in the face-to-face interview findings:

1. Students have a lack of clarity in the roles of other disciplines.

2. Health policy changes strengthened teaching philosophy due to foreseen positive outcomes.

3. Health policy changes have not made an impact.

4. Health policy changes affected teaching philosophy due to stricter modifications.

5. There are pre-conceived notions among agency staff regarding students' knowledge deficit of policies.

6. Lack of cooperation and willingness from students to use their own resources.

7. Complacency of nursing staff in guiding students in cost-effectiveness.

8. Educators need to acquire new knowledge and creative teaching methods to teach cost-effectiveness.

9. Students learn QSEN competencies in Foundations.

10. Students eventually become familiar with rules, regulations, and duties.

11. Research is prerequisite to learning various interprofessional roles.

12. IPC helps in appreciating all the disciplines and increases drive to teach.

13. IPC helps provide guidelines and support of skill training.

\section{Emerging Themes from Participant-Observations}

The findings of the data analysis of participant-observations led to the emergence of the following themes:

1. There were no discussions or evidence of limited budget issues. It was not a significant barrier for nurse educators to teach students to practice in a cost-controlled healthcare environment.

2. Nurse educators gave students the freedom and independence to determine the roles of the different professionals in the healthcare system.

3. Nurse educators emphasized the importance of collaborative teamwork and exposed students to an interprofessional collaborative practice environment.

\section{Data Triangulation}

Triangulating data from multiple sources of evidence essentially provides multiple measures of the same phenomenon. Presentation of the triangulated results was organized relevant to answering the four research sub-questions. 


\section{Triangulated Findings for Central Research Question}

How do nurse educators in a pre-licensure undergraduate baccalaureatenursing program describe their role in preparing students for interprofessional collaborative care practices? Findings indicated the majority of nurse educators describe their role in preparing students for interprofessional collaborative practice using classroom, clinical and simulation teaching strategies. Clinical environments were most often described as settings in which students gain the most knowledge through actual participation with the interprofessional team.

\section{Triangulated Findings for Research Sub-question 1}

How do these nurse educators incorporate the impact of health policy and government regulations in their teaching philosophy? The prevailing theme that emerged from the triangulated data was healthcare policies have always been included in teaching policy; thus, no change was described in teaching philosophy.

\section{Triangulated Findings for Research Sub-question 2}

How do these nurse educators describe the barriers to teaching students to practice in a cost controlled healthcare environment? There were different perspectives on cost-control and descriptions of healthcare policy compliance in teaching facilities. Survey findings indicated that lack of state funding to healthcare and higher education was a barrier to teaching in the classroom setting. However, through triangulation of multiple data sources, the predominate theme was that a cost-controlled clinical environment was not described as a barrier to teaching.

\section{Triangulated Findings for Research Sub-question 3}

How do these nurse educators teach students to understand their unique professional role and the role of other disciplines in the care of their patients? The prevailing theme was nurse educators teach their students to understand their unique professional roles as safe, patient-centered caregivers of quality, evidence-based nursing care; and the roles of other disciplines while experiencing and participating in nursing care during clinical rotations. Nurse educators described how students participated in interprofessional collaboration, learning the value of an interprofessional healthcare environment. Before triangulation, the first major theme to emerge was students do not learn QSEN and AACN competencies in this specific nursing education program (11 out of 16 participants, $69 \%$ ). 


\section{Triangulated Findings for Research Sub-question 4}

How has the current interprofessional healthcare environment changed the lives of these nurse educators? The main finding was that an interprofessional healthcare environment has been integrated in the professional lives of nurses. Nurse educators have taught the concepts of interprofessional healthcare for years, (comparing IPC to discharge planning) therefore, there was no change reported in the lives of these nurse educators.

\section{Discussion and Recommendations}

The research study developed a better understanding of the phenomena of interprofessional collaboration among nurse educators. Although the major themes identified were the primary focus for answering the research questions, several minor themes emerged and one is worth discussing.

Face-to-face interview findings for interview protocol question 9 were surprising. Do students learn QSEN or AACN (IPEC) competencies? If so, at what level? Findings indicated $69 \%$ of nurse educators interviewed answered "no". After triangulation of all data sources (survey, interviews and observations), the overarching theme was that students learn IPC during clinical experiences. During further probing questions, educators seemed to explain they taught teamwork and the importance of communication and collaboration with team members. There was evidence of electronic charting which complied with the fourth AACN (IPEC) competency. Apparently, as reported, educators are teaching concepts of IPC in an informal style. There was no evidence that educators formerly introduce national guidelines (QSEN and AACN competencies).

\section{Discussion of Findings in Relation to the Literature}

Understanding the perspectives of nurse educators regarding IPC is crucial in developing learning opportunities for graduate baccalaureate students to practice effectively in today's healthcare environment. According to the JMJF (2013), IPEC is working with health professions including medicine, dentistry, nursing, social sciences, pharmacy, and allied health to refine educational content for interprofessional teams. Opportunities envisioned will allow health profession students to interact and learn with those outside their profession with the common goal of "building a safer and better patient-centered and community-population oriented US healthcare system" (IPEC 2011). Embedded in current healthcare and nursing education reform literature are common themes related to competencies (IPEC 2011).

Findings from this case study indicate clinical agencies, hospitals and other healthcare professionals are engaged in interprofessional care practice. Nurse educator participants were aware of the concept, and understood the meaning of IPC. According to respondents, there are no formal curriculum 
guidelines for QSEN or AACN competencies. Participants were aware of the QSEN initiative, but unfamiliar with AACN or IPEC competencies Teamwork and collaboration (one of the QSEN competencies) were taught; however not in the QSEN competency framework. Interprofessional communication and interprofessional teamwork (two of the AACN [IPEC] competency domains) were described as being taught, but not in the AACN (IPEC competencies) context. It appeared the educators were assigning students to practice settings that incorporate IPC. However, educators had a difficult time describing IPC based on recent national initiatives in the practice environment.

There is a sense of urgency for all healthcare professions to implement interprofessional collaboration (IPC), interprofessional education (IPE) and interprofessional collaborative practice (ICP) in a patient-centered healthcare environment. With a lack of standards, outcomes are difficult to measure (O'Brien 2013). The ACA caused healthcare providers and policy makers to realize with shortages of primary care physician and nurses, increased collaboration, and teamwork among health professions is necessary to provide care for an increasingly aging population with multiple comorbid chronic conditions.

Findings from the research indicated hospitals are complying with new federal regulations. According to respondents, educators did not find a costcontrolled environment a barrier to teaching. Decreased state funding for medical care, higher education, and faculty salaries were noted to be an underlying complaint, but overall, educators did not seem to let these issues act as barriers to teaching.

Another finding relevant to new health policy changing teaching philosophies is that participants described their philosophies and teaching strategies unchanged from previous years. Respondents described the healthcare environment essentially the same, with just new terms to describe what nurses have always practiced. Younger faculty seemed to be interested and open to new concepts. One participant was interested in acquiring resources for teaching strategies to promote IPC.

\section{Relationship between the Findings and the Theoretical Framework}

Research findings associated with understanding how nurse educators in a prelicensure undergraduate baccalaureate-nursing program describe their role in preparing students for interprofessional collaborative care practices were informed and supported by a blended theoretical framework. The current transformation of health professions education is complex, deliberate, and policy informed. Therefore, this case study's advocacy-liberatory framework blended elements of complexity theory and transformational learning theory with RTBC. Current research identifies nursing as complex, and healthcare as a complex system (Sargeant 2009). Application of complexity theory provides nurse educators the opportunity to view nursing education from a different perspective as it moves from an individual discipline to an interprofessional 
approach in response to education, the health team, health system, and environment (Sargeant 2009).

Study participants shared conducive learning situations, especially in clinical settings. The health system environments were policy driven and complex. They described clinical settings that related to RTBC platform. Clinical settings and bureaucratic cultures required nursing management to collaborate with health professionals to obtain the best outcomes, the highest quality care, with the shortest length of stay. Transformational learning and bureaucratic caring theory conceptualize and broaden an understanding of health policy reform as socio-cultural, organizational, political, and economic change. Participants applied transformative teaching-learning strategies with developmental learning techniques. Integrating RTBC offers alternative perspectives to existing nursing education teaching strategies, highlighting organizational cultures and complex caring, thereby, encouraging educators to think differently about interprofessional collaboration.

Sargeant (2009) best describes findings of this research study, "transformative learning changes the way we see ourselves, those around us, and the world. It is not simply learning new knowledge, skills, or extending what we already know into a new topic or domain. It actually changes what we know" (p. 182).

\section{Limitations}

A self-reporting online survey, AACN-CFNESI2 was used for initial data collection. Limitations of this type survey were that all potential participants may not complete the survey, may or may not be truthful in responding, or may not understand the survey questions. The small number of respondents to the online survey was a limitation in this study. Although the number of respondents was sufficient to answer the research question, it was not a solid representation of all nurse educators teaching in the baccalaureate-nursing program. Another limitation was the time constraints. If more time had been allotted, a larger number of participants may have participated and more observations made.

\section{Implication of the Findings for Practice}

The research questions aligned with rapid changes taking place in healthcare redesign. The sub-questions were designed to stimulate more discussion concerning background issues. Nurse educators must continue to learn and develop new teaching strategies to promote interprofessional learning initiatives. Transferability was established by conducting research that may be easily transferable as it represents a small sample of nurse faculty facing a national teaching dilemma in nursing education. The research provided enough information to be transferred by readers to determine whether this study's findings are applicable to a new situation. This research showed the participants have the knowledge, experience, or expertise necessary to provide 
information that the nursing discipline would find meaningful regarding interprofessional collaboration. Helping nurse educators to understand the changing role they face, and how they teach in an ever-changing healthcare environment was an objective of the study.

\section{Recommendations for Further Research}

The Carnegie Foundation Report (2010) acknowledged the profound changes in nursing practice, recommending changes in educating nurses and nurse educators. Collaboration among interdisciplinary professionals is vital to collaborative healthcare. Nursing faculty is responsible for preparing student nurses as leaders and patient advocates in collaborative healthcare settings. Further research is needed to provide guidance on how health professionals can collaborate with each other. New research will help develop strategies and interventions used in teaching-learning environments, thereby helping students become competent collaborative practitioners (D'Amour and Oandasan 2005).

The JMJF is dedicated to improving health professional education. The foundation recommended incorporating educational reform and practice redesign, emphasizing they not be developed in isolation (JMJF 2013). The study's findings indicate a need for further research to understand the needs of baccalaureate nursing program faculty to conform successfully to national nursing regulatory initiatives.

There seems to be a need to understand new policy terminology and how it applies to teaching-leaning methods and, or replaces old policy terminology. Further research on curriculum reform to meet the needs of healthcare reform was also identified. One case study cannot answer all questions and uncover all concepts of a phenomenon. A recommendation based on this study is to compare and contrast responses of two different baccalaureate programs in relation to IPC preparation and curriculum reform. Another recommendation is to study new graduate perceptions of student preparation for IPC.

\section{Conclusions}

The purpose of this qualitative case study was to learn how nurse educators in an undergraduate baccalaureate-nursing program describe their role in preparing students for interprofessional collaborative care practices. The research priority in this research study was to seek believability. Verification was assumed through cohesive, insightful, trustworthy qualitative research processes and case study protocol. Yin $(2009,2011)$ provided the design and methodological model. A blended theoretical framework was used to relate a nursing practice phenomenon to a nursing education phenomenon through the lens of RBTC, complexity theory and transformational learning theory.

The central research question was answered using multiple data sources. Construct validity was addressed by triangulating multiple data sources of evidence which led to converging lines of inquiry. 
Findings of the research indicated the majority of nurse educators describe their role in preparing students for interprofessional collaborative practice using classroom, clinical and simulation teaching strategies. Clinical environments were most often described as settings in which students gain the most knowledge through actual participation with the interprofessional team. Currently, this nursing program's curriculum does not include IPE learning activities with other healthcare professionals.

Although standards are beginning to be set, much research and faculty development is needed for nurse educators to incorporate interprofessional collaboration in teaching plans and strategies. There is an opportunity for the nursing profession to be front-runners in promoting the global initiative of interprofessional collaboration.

\section{References}

Alberto J, Herth K (2009) Interprofessional collaboration within faculty roles: teaching, service, and research. Online Journal of Issues in Nursing 14(2): 1G$16 \mathrm{G}$.

AACN-American Association of Colleges of Nursing (2002) Hallmarks of the professional nursing practice environment. Retrieved from http://goo.gl/Cxx1tC.

AACN-American Association of Colleges of Nursing (2005) Faculty shortages in baccalaureate and graduate nursing programs: Scope of the problem and strategies for expanding the supply. Retrieved from http://goo.gl/KeXEfV.

Benner P, Sutphen M, Leonard V, Day L (2009) Educating Nurses: A Call for Radical Transformation. San Francisco, CA: Jossey Bass.

Carnegie Foundation (2010) Educating nurses: A call for radical transformation. Retrieved from http://goo.gl/pcfK21.

Cronenwett L, Sherwood G, Barnsteiner J, Disch J, Johnson J, Mitchell P, et al. (2007) Quality and safety education for nurses. Nursing Outlook 55(3): 122-131.

D'amour D, Oandasan I (2005). Interprofessionality as the field of interprofessional practice and interprofessional education: an emerging concept. Journal of Interprofessional Care 198(20).

Didion J, Kozy M, Koffel C, Oneail K (2013) Academic/clinical partnership and collaboration in quality and safety education for nurses education. Journal of Professional Nursing (29)2: 88-94.

Garity J (2005) Ethics: Relationship of the ANA code of ethics to nurses' collaborative efforts. Online Journal of Issues in Nursing 10(3).

Hidalgokehoe MA (2012) Complexity theory and nursing: explanation and application. Retrieved from http://goo.gl/VXYTRO.

IHI-Institute for Healthcare Improvement (2015) IHI Triple Aim initiative. Retrieved from http://goo.gl/BvCeex.

IOM-Institute of Medicine (1972) Educating for the health team. Retrieved from https://goo.gl/VlklSQ.

IOM-Institute of Medicine (2003) Health professions education: A bridge to quality. Washington, DC: National Academies Press.

IOM-Institute of Medicine (2010) The future of nursing: Leading change, advancing health. Retrieved from http://goo.gl/V8ch8I. 
IPEC-Interprofessional Education Collaborative (2011) Core competencies for interprofessional collaborative practice: Report of an expert panel. Washington, DC: IPEC.

JMJF-Josiah Macy Jr. Foundation (2013) Transforming patient care: aligning interprofessional education with clinical practice redesign. Conference Recommendations. Atlanta, GA. Retrieved from http://goo.gl/uoGlcs.

Lodico MG, Spaulding DT, Voegtle KH (2010) Methods in educational research from theory to practice (2nd edn.). San Francisco, CA: Jossey-Bass.

Merriam SB, Caffarella RS, Baumgartner LM (2007) Learning in adulthood: A comprehensive guide. San Francisco, CA: Jossey-Bass.

NLN-National League of Nursing (2003) Innovation in nursing education: A call to reform. Retrieved from http://goo.gl/8fsBZY

O'Brien J (2013) Interprofessional collaboration. AMN Healthcare Education Services. Retrieved from http://goo.gl/XX9htT

Patient Protection and Affordable Care Act (2010) Patient Protection and Affordable Care Act Detailed Summary. Retrieved from http://goo.gl/dETs6.

QSR International Pty Ltd. (2010) NVivo (Version 9) [Qualitative data analysis software].

Ray MA (1998) Complexity and nursing science. Nursing Science Quarterly (11)3: 91-93.

Sargeant J (2009) Theories to aid understanding and implementation of interprofessional education. Journal of Continuing Education in the Health Professions 29(3): 178-184.

Schmitt M (2011) Core competencies for interprofessional collaborative practice. Retrieved from http://goo.gl/hSE6lE

Stokowski LA (2011) Overhauling nursing education. Medscape Nurses - Nursing Perspectives. Retrieved from http://goo.gl/LrOiRJ

Thannhauser J, Russell-Mayhew S, Scott C (2010) Measures of interprofessional education and collaboration. Journal of Interprofessional Care 24(4): 336-349.

Tri-Council for Nursing (2010) Statement in support of the IOM future of nursing report. Retrieved from http://goo.gl/6FUEUE.

Turkel MA, Ray MA, Kornblatt L (2012). Instead of reconceptualizing the nursing process: Let's rename it. Nursing Science Quarterly 25(2): 194-198.

Varkey P, Reller MK, Smith A, Ponto J, Osborn M (2006) An experiential interdisciplinary quality improvement education initiative. American Journal of Medical Quality 21(5): 317-322.

WHO-World Health Organization (2010) Framework for action on interprofessional education and collaborative practice. Retrieved from http://goo.gl/PSk1Oz

Yin RK (2009) Case study research: Design and methods (4th edn.). Thousand Oaks, CA. Sage Publications, Inc.

Yin RK (2011) Qualitative research from start to finish. New York, NY. The Guilford Press. 
